\title{
Veno-venous extracorporeal membrane oxygenation successfully treated a case of severe pulmonary hemorrhage caused by leptospirosis
}

\author{
H. J. Wang* (D, G. Z. Chen, C. J. Zhou, Y. FU and L. N. YAO
}

\begin{abstract}
Background: Pulmonary hemorrhage is an important complication of leptospirosis. Once acute respiratory distress syndrome (ARDS) occurs as a secondary condition, treatment is extremely difficult and the mortality rate is very high.

Case presentation: The patient was a 49-year-old. He was admitted to the hospital because he had experienced a fever and cough for 4 days. Hemorrhage, respiratory failure, ARDS and other symptoms appeared soon after admission. Due to severe pulmonary hemorrhage secondary to ARDS, mechanical ventilation was performed through tracheal intubation. During intubation, the patient suffered cardiac arrest, and the patient's condition worsened. He was confirmed to have leptospirosis through second-generation sequencing of the alveolar lavage fluid. Finally, we successfully treated the patient with penicillin as an anti-infective medication and venous-venous extracorporeal membrane oxygenation ( $\mathrm{v}-\mathrm{vECMO}$ ). To the best of our knowledge, this report is the first to describe the successful application of ECMO in mainland China.

Conclusions: Leptospirosis can induce serious but transient ARDS with a better prognosis than other causes of ARDS. Our patient was successfully treated with V-vECMO.
\end{abstract}

Keywords: Leptospirosis, Pulmonary hemorrhage, ARDS, ECMO, Case report

\section{Background}

Leptospirosis is a zoonotic infectious disease caused by Leptospira. Leptospiral infection has a broad spectrum of clinical manifestations, ranging from a subclinical or mild illness to a fulminant life-threatening illness. Symptoms suggesting leptospirosis are non-specific but usually include fever, headache and myalgia, sometimes with hemorrhage or meningitis as the initial presentation $[1,2]$, and the disease has an average mortality rate of

\footnotetext{
* Correspondence: whj269696@163.com

Department of Intensive Care Unit, the Affiliated People's Hospital of Ningbo University, 251 East Baizhang Road, Ningbo City 315010, Zhejiang Province, P.R. China
}

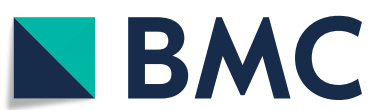

(c) The Author(s). 2020 Open Access This article is licensed under a Creative Commons Attribution 4.0 International License, which permits use, sharing, adaptation, distribution and reproduction in any medium or format, as long as you give appropriate credit to the original author(s) and the source, provide a link to the Creative Commons licence, and indicate if changes were made. The images or other third party material in this article are included in the article's Creative Commons licence, unless indicated otherwise in a credit line to the material. If material is not included in the article's Creative Commons licence and your intended use is not permitted by statutory regulation or exceeds the permitted use, you will need to obtain permission directly from the copyright holder. To view a copy of this licence, visit http://creativecommons.org/licenses/by/4.0/ The Creative Commons Public Domain Dedication waiver (http://creativecommons.org/publicdomain/zero/1.0/) applies to the data made available in this article, unless otherwise stated in a credit line to the data. hemorrhage secondary to acute respiratory distress syndrome (ARDS), the mortality rate increases to 51 to $100 \%$ due to the lack of effective treatment $[3,4]$. Use ECMO as a potential treatment modality with leptospirosis is increasing. However, this method requires advanced technology and is expensive. Currently, case reports are scarce and have not been widely conducted, particularly in developing countries. To our knowledge, no similar studies have been conducted in mainland China, particularly in patients with secondary cardiac arrest. The report of the successful treatment of secondary cardiac arrest induced by severe intravenous 
hemorrhagic leptospirosis with $\mathrm{v}$-vECMO in our hospital is described below.

\section{Case presentation}

The patient was a 49-year-old self-employed male in good health who liked to tend to flowers and plants and was found to show evidence of mouse bites. The patient developed a cough at home 4 days prior to admission. Occasionally, the sputum was tinged with red blood, which was accompanied by pharyngeal and knee pain; his highest temperature was $39.2^{\circ} \mathrm{C}$ and was accompanied by chills. He did not experience night sweats, herpes of the mouth, chest pain, abdominal pain, diarrhea, hoarseness, headache, vomiting, etc. He visited our hospital because of chest tightness after activity. Upon admission, the patient was conscious, with a temperature of $37.5^{\circ} \mathrm{C}$, a pulse rate of 105 beats $/ \mathrm{min}$, a respiration rate of 18 breaths/min, and a blood pressure of 100/49 $\mathrm{mmHg}$. The following observations were recorded: the bulbous conjunctiva was free of congestion, his lips were not bluish, wet rales were heard in both lungs, his heart rate was 105 beats/minute. The laboratory tests showed a c-reactive protein level of $90.87 \mathrm{mg} / \mathrm{L}$, and white blood cell count, $9.6 \times 10^{9} / \mathrm{L}$; hemoglobin, $117 \mathrm{~g} / \mathrm{L}$,platelet count, $96 \times 10^{9} / \mathrm{L}$. The results of the blood gas analysis revealed a $\mathrm{PO}_{2}$ of $119 \mathrm{mmHg}, \mathrm{pH}$ of 7.43; $\mathrm{PCO}_{2}$ of 38 $\mathrm{mmHg}$, and procalcitonin (PCT) level of $4 \mathrm{pg} / \mathrm{mL}$ (Table 1). The chest CT revealed bilateral pulmonary infiltrations (Fig. 1). After admission, piperacillin tazobactam was administered at a dose of $4.5 \mathrm{~g}$ iv q8h due to moderate severe pneumonia, a $40 \mathrm{mg}$ methylprednisolone injection was administered to control body temperature, and supportive nasal catheter oxygenation was provided $(5 \mathrm{~L} / \mathrm{min})$. The patient's condition worsened after $4 \mathrm{~h}$, with a temperature increased to $39^{\circ} \mathrm{C}$, a heart rate of 150 beats/min, a respiratory rate of 26 breaths/min, a blood pressure of $85 / 55 \mathrm{mmHg}$, shortness of breath, but no chills; additionally, the patient's $\mathrm{SpO}_{2}$ level through the nasal catheter decreased to $70 \%$. The patient's $\mathrm{SpO}_{2}$ increased to $86 \%$, when switched to a venturi mask, but his shortness of breath persisted. The patient was transferred to ICU for adequate fluid resuscitation and blood culture is collected. The patient soon exhibited hemoptysis, his $\mathrm{SpO}_{2}$ decreased to $67 \%$, the $\mathrm{PO}_{2}$ of $43 \mathrm{mmHg}$, blood pressure was $96 / 56 \mathrm{mmHg}$, and wet rales in both lungs had significantly increased. A pulse index continuous cardiac output examination was performed, which resulted in a extravascular lung water index of $36.9 \mathrm{~mL} / \mathrm{kg}$ (normal range 3.0-7.0) and pulmonary vascular permeability index of $6.0 \mathrm{dn} . \mathrm{s} . \mathrm{m}^{2} / \mathrm{cm}^{5}$ (normal range 1.0-3.0). The patient's heart function was normal, his lung permeability increased, and the presence of extravascular fluid in the lung increased. After $10 \mathrm{~h}$ in the ICU, the patient's condition deteriorated again. He was agitated under noninvasive mechanical ventilation, and the $\mathrm{SpO}_{2}$ level was difficult to maintain within the normal range. Mechanical ventilation via tracheal intubation was performed. During the process of endotracheal intubation, a large amount of bloody secretions sprouted from the airway, the heart rate rapidly decreased to 40 beats $/ \mathrm{min}$, and the arterial pulse and $\mathrm{SpO}_{2}$ were not detected; Cardiopulmonary resuscitation was implemented immediately. After 1 min of cardiopulmonary resuscitation, the patient recovered spontaneous circulation but was still agitated and in respiratory distress with an $\mathrm{SpO}_{2}$ of $80 \%$, despite full mechanical ventilator support. Five hours after the patient was sedated using midazolam and fentanyl through continuous micropumping, his shortness of breath was still not relieved. The ventilator provided a small tidal volume

Table 1 Laboratory examination results during ICU hospitalization

\begin{tabular}{|c|c|c|c|c|c|c|c|c|c|c|c|c|c|}
\hline Days & Normal range & 0 & 1 & 2 & 3 & 4 & 5 & 6 & 7 & 8 & 9 & 10 & 11 \\
\hline WBCX109/L & $3.5-9.5$ & 6.4 & 7.8 & 10.3 & 9.7 & 9.9 & 11.7 & 14.2 & 20.1 & 23.3 & 25.3 & 30.7 & 20 \\
\hline hemoglobin ( $\mathrm{g} / \mathrm{L}$ ) & $115-150$ & 117 & 64 & 90 & 102 & 81 & 93 & 99 & 121 & 118 & 117 & 128 & 127 \\
\hline platelet (× 109/L) & $125-350$ & 96 & 57 & 102 & 118 & 170 & 170 & 197 & 164 & 174 & 186 & 231 & 250 \\
\hline PCT (ng/mL) & $0.02-0.05$ & 4.7 & 74 & 29 & 12.8 & 12.8 & 3.28 & 1.96 & 0.95 & 0.62 & 0.57 & 0.4 & 0.22 \\
\hline CRP (mg/dL) & $0-8.0$ & 93 & 119.6 & 119 & 23 & 16 & 14 & 6.69 & 4.07 & 6.31 & 3.51 & 26.14 & 53.2 \\
\hline CREA (umol/L) & $41-81$ & 90 & 108 & 79 & 87 & 62 & 54 & 46 & 42 & 46 & 42 & 39 & 48 \\
\hline AST(u/L) & $13-35$ & 26 & 24 & 25 & 28 & 27 & 43 & 51 & 62 & 38 & 28 & 16 & 14 \\
\hline $\operatorname{ALT}(\mathrm{u} / \mathrm{L})$ & $7-40$ & 98 & 93 & 115 & 132 & 150 & 169 & 173 & 135 & 73 & 52 & 37 & 38 \\
\hline TBIL (umol/L) & $3.1-17.1$ & 14.1 & 41.3 & 34.7 & 29 & 20.3 & 27.7 & 24.2 & 21.2 & 19.6 & 17.6 & 12.3 & 14.3 \\
\hline PT (s) & $9.2-13.1$ & 14.4 & 29.4 & 18.4 & 19.3 & 23.5 & 31.3 & 140 & 16.6 & 14.8 & 12.7 & 16.5 & 13.1 \\
\hline APTT (s) & $25.4-40.9$ & 27.5 & 28.9 & 61.5 & 40.4 & 46.4 & 38.4 & 38.4 & 33.3 & 32.6 & 32.1 & 27.5 & 26.9 \\
\hline INR & $0.82-1.15$ & 1.1 & 1 & 1.12 & 1.11 & 1.14 & 1.09 & 1.14 & 1.23 & 1.17 & 1.1 & 1.01 & 1.14 \\
\hline FIB (g/l) & $2.0-4.0$ & 1.93 & 1.94 & 1.66 & 1.74 & 1.96 & 1.83 & 2.4 & 2.92 & 2,43 & 1.93 & 3.4 & 3.7 \\
\hline
\end{tabular}




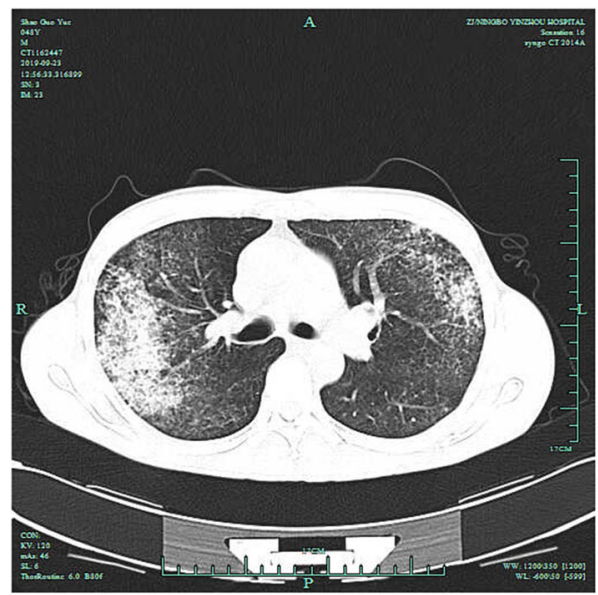

a

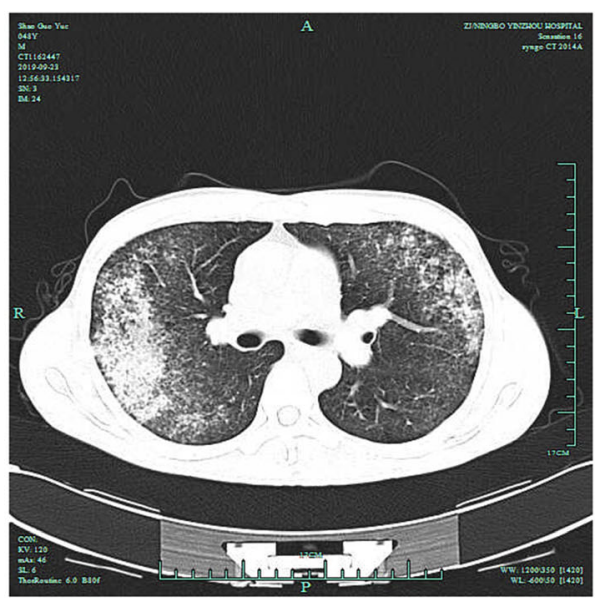

b

Fig. 1 Chest CT on the day of admission. Both lungs presented exudative lesions, which are obvious in the image of the right lung

ventilation with a frequency of $50-60$ breaths $/ \mathrm{min}$. The blood gas analysis indicated a $\mathrm{pH}$ of 7.19, $\mathrm{pCO}_{2}$ of 65 $\mathrm{mmHg}, \mathrm{PO}_{2}$ of $61 \mathrm{mmHg}$, and hemoglobin concentration of $68 \mathrm{~g} / \mathrm{dL}$. The patient showed significant pulmonary hemorrhage and clinical manifestations of ARDS. After a discussion among the staff of the cardiology department, thoracic surgery department, ICU, respiratory department and other experts in our hospital, v-vECMO was initiated at $4.5 \mathrm{~L} / \mathrm{min}$, with sweeping gas flow through the oxygenator at $4.5 \mathrm{~L} / \mathrm{min}$ of $100 \%$ oxygen. After the procedure, the patient's $\mathrm{SpO}_{2}$ was $100 \%$. The partial thromboplastin time (PTT) was maintained at $40-50 \mathrm{~s}$ by administering a heparin infusion, and the PTT was monitored every $4 \mathrm{~h}$ to avoid aggravating the pulmonary hemorrhage. The ECMO blood flow was maintained at $4.5 \mathrm{~L} / \mathrm{min}$ during the first 3 days. Because general community-acquired pneumonia was considered an unlikely diagnosis, $4.5 \mathrm{~g}$ of piperacillin tazobactam iv $\mathrm{q} 8 \mathrm{~h}$ and $0.5 \mathrm{~g}$ of azithromycin iv $\mathrm{qd}$ as anti-infection medications and $40 \mathrm{mg}$ of methylprednisolone $\mathrm{q} 8 \mathrm{~h}$ were continued. A tracheoscopic examination prompted diffuse bleeding in the airway and alveolar lavage fluid was collected and for second-generation gene sequencing on the next day. The hemoglobin concentration decreased to $61 \mathrm{~g} / \mathrm{dL}$ after an infusion with 3 units of red blood cells, and the platelet count decreased to $57 \times 10^{9} / \mathrm{L}$. Six units of a red blood cell suspension were applied to correct the anemia, and 10 units of platelets and $600 \mathrm{~mL}$ of fresh frozen plasma were re-administered to supplement the clotting factors. The laboratory tests revealed an increase in the PCT level to $77 \mathrm{ng} / \mathrm{mL}$, and the antibiotics were upgraded to $0.5 \mathrm{~g}$ of intravenous meropenem q6h for a suspected severe infection. Four days later, secondgeneration gene sequencing of the patient's alveolar lavage fluid was positive for Leptospira interrogans.
Although the blood culture and urine culture did not detect leptospirosis, prior to discharge, the results showing strongly positive Leptospira serology with an IgM titer of 1:1280 detected using an enzyme-linked immunosorbent assay (ELISA). Leptospirosis was considered, and the patient was administered an injection of $800,000 \mathrm{U}$ of penicillin $\mathrm{q} 8 \mathrm{~h}$ (with a first dose of $400,000 \mathrm{U}$ ) as antiinfective treatment. The patient was sedated using midazolam and fentanyl during the ECMO supportive treatment, and no obvious complications occurred. After 6 days, the chest radiograph of the patient was significantly improved and ECMO was stopped (Fig. 2). On day 9, the endotracheal tube was removed, and the patient was transferred to the general ward on day 10.

\section{Discussion and conclusions}

Leptospirosis is an animal-transmitted infectious disease that was once widely prevalent in Zhejiang Province. From the mid-1960s to the early 1970s, four great epidemics occurred. After the mid-1970s, the incidence of leptospirosis decreased annually. Since 2000, the annual incidence in the whole province has decreased to less than $0.2 / 100,000$. Some medical personnel have an insufficient understanding of leptospirosis, and the etiology is often difficult to determine, which might easily lead to a missed diagnosis and misdiagnosis. Our medical institution has not diagnosed leptospirosis for more than 20 years, and the relevant laboratory tests have not been routinely performed. The clinical manifestations of leptospirosis vary and include fever, jaundice and acute renal injury. Lung manifestations occur in $20-70 \%$ of patients, but are often masked by other symptoms. Severe pulmonary hemorrhage is the main cause of death associated with leptospirosis and usually occurs in 4-6 days; severe pulmonary hemorrhage manifests as extensive 


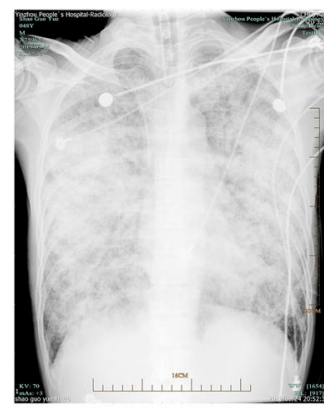

a

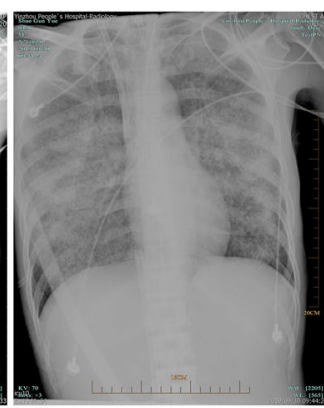

b

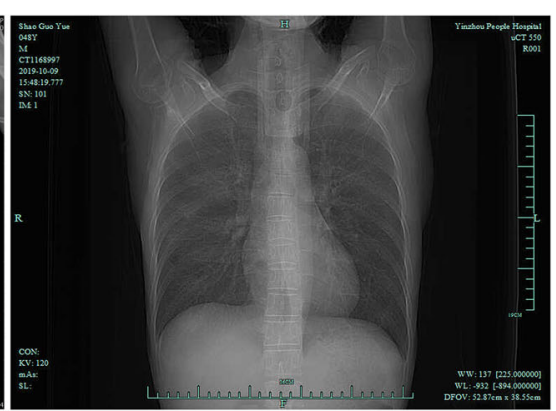

C

Fig. 2 a: Chest X-ray before ECMO treatment. Diffuse exudation was observed in both lungs, presenting as a large white lung. $\mathbf{b}$ : Chest $X$-ray on day 6 of ECMO treatment. The diffuse exudation in both lungs was significantly absorbed compared with the image captured at admission. $\mathbf{c}$ Chest X-ray on the 9th day after the removal of ECMO. The pneumonia was completely absorbed without residual disease

interstitial and alveolar hemorrhage, pulmonary congestion, and pulmonary edema. The specific mechanism is unknown. According to Croda et al, the linear deposition of immunoglobulins (IgA, IgG, and IgM) and complement on the alveolar surface may play a role in the pathogenesis [5]. Researchers have posited that a Jarisch-Herxheimer reaction after the first application of antibiotics may be the main cause of disease progression and it particularly aggravates pulmonary hemorrhage [6]. This patient also showed a rapid onset of symptoms $4 \mathrm{~h}$ after the application of antibiotics on the fourth day of onset, which was accompanied by severe pulmonary hemorrhage, shock, hyperthermia, etc. The determination of whether the disease itself progresses or if the Jarisch-Herxheimer reaction that occurs after the application of antibiotics causes adverse effects remains a challenge; with the exception of fever, shock is similar to the disease itself, and no specific and objective laboratory evidence is available. This patient had no obvious clinical manifestations of chills. Clinicians have not determined whether the lack of this manifestation is related to the use of methylprednisolone at admission. Some clinicians postulate that hormones prevent the occurrence of the Jarisch-Herxheimer reaction [7]. However, the PCT dramatically increased from a low level within $24 \mathrm{~h}$ of the patient's deterioration and decreased rapidly the next day, which is rare in patients with sepsis. Observations of additional cases are required to determine whether this finding is related to the fact that the Jarisch-Herxheimer reaction is a self-limited response.

Pulmonary hemorrhage is a serious complication of leptospirosis. The accumulation of hemorrhagic fluid in the alveoli can lead to airway obstruction, loss of ventilated blood and acute gas exchange disorders, which may cause patients to experience significant difficulty breathing. Acute respiratory distress includes hypoxemia; despite the timely initiation of conventional mechanical ventilation, hypoxemia may still be difficult to treat and has a high mortality rate $[4,8]$. Although some studies have attempted to treat this disease with high doses of hormones, plasma exchange, hemostatic drugs, high-frequency oscillating ventilation and other methods, the effects remain unclear $[9,10]$. Despite the report of a successful ECMO application in patients with ARDS [11], few studies have examined whether ECMO is useful for ARDS caused by severe pulmonary hemorrhage in individuals with leptospirosis; conclusive evidence is lacking, particularly while maintaining ECMO, regarding whether the high-intensity anticoagulation required to avoid embolization events will aggravate pulmonary hemorrhage, and this factor is currently the most important concern. Overall, active hemorrhage is still a relative contraindication for ECMO application. In the present study, the patient presented obvious pulmonary hemoptysis and a reduced hemoglobin concentration after admission, and the diagnosis was unclear. The prediction of whether anticoagulation medication during ECMO maintenance would lead to uncontrolled pulmonary hemorrhage was difficult to determine. However, the patient still presented with significant dyspnea symptoms under high-level mechanical ventilation, which was an important reason why the patient required ECMO. Although heparin should be avoided as much as possible during ECMO maintenance in patients with pulmonary hemorrhage [12], we adjusted the anticoagulant intensity to maintain an PTT of 40-50 s and simultaneously injected plasma, platelets, erythrocyte suspensions and other supportive treatment measures. Fortunately, uncontrolled bleeding was not observed during ECMO in this patient. Despite invasive ventilation with a high PEEP, a maximum oxygen supply and the use of muscle relaxants, analgesics and sedatives, the patient was still difficult to treat. V-vECMO was used to temporarily replace the lung function of the patient and adopt protective strategies to avoid ventilator-related 
lung injury. After 6 days, the treatment was successful, suggesting that as long as the primary disease is treated effectively, heparin is a feasible treatment to maintain the coagulation status in a reasonable range that does not aggravate pulmonary hemorrhage. ECMO allows the patient's lungs to rest adequately, which effectively facilitates treatment. This effect may be related to the fact that respiratory failure caused by leptospirosis-induced hemorrhaging is simpler and more transient than the condition caused by other diseases [13].

In conclusion, pulmonary haemorrhage is a serious complication of leptospirosis. In recent years, the incidence of leptospirosis has increased. In this article, we confirmed that genetic sequencing of alveolar lavage fluid also effectively diagnoses the disease, thereby avoiding a missed diagnosis due to negative blood culture, urine culture, and sputum culture. This disease has a high mortality rate, and very limited treatment methods are available. With the increasing number of reports of successful treatment using ECMO, this method may be an effective measure to reduce the mortality associated with leptospirosis; however, further prospective largescale controlled experimental studies are needed to confirm these findings. Finally, this article has some limitations. For example, because this study reports a retrospective analysis and is limited by cost, blood and urine cultures were not further sequenced after blood and urine cultures were negative.

\section{Abbreviations}

ARDS: Acute respiratory distress syndrome; v-vECMO : Venous-venous extracorporeal membrane oxygenation; PCT: Procalcitonin; ICU: Intensive care unit

\section{Acknowledgments}

We would like to thank the patient for agreeing to the publication of this article and all the doctors and staff who assisted us with this report. We are also very grateful to the other translators and indirect tutors.

\section{Authors' contributions}

$\mathrm{HJ}$ participated in the case report, performed the main practical work, data analysis and drafted the manuscript. GC was responsible for preparing the paper and provided the final approval. CZ was responsible for the acquisition, analysis, or interpretation of data and revised the paper critically for important intellectual content. FY and YLN were actively participated in diagnosis, treatment, and responsible for polishing the English translation. All authors have read and approved the manuscript.

\section{Funding}

No funding was received for this study.

\section{Availability of data and materials}

All data have been presented within the manuscript and in the form of images.

\section{Ethics approval and consent to participate}

All procedures performed in studies involving human participants were performed in accordance with the ethical standards of the Ethics Committee of Ningbo Yinzhou People's Hospital and the 1964 Declaration of Helsinki and its later amendments or comparable ethical standards. Informed consent was obtained from the patient.

\section{Consent for publication}

Written informed consent was obtained from the patient for publication of this case report and any accompanying images. A copy of the written consent form is available for review by the Editor-in-Chief of this journal.

\section{Competing interests}

We declare have no competing interests to declare.

Received: 26 December 2019 Accepted: 16 October 2020

Published online: 27 October 2020

\section{References}

1. Puca E, Pilaca A, Kalo T, Pipero P, Bino S, Hysenaj Z, Abazaj E, Gega A, Petrela E, Kraja D. Ocular and cutaneous manifestation of leptospirosis acquired in Albania: a retrospective analysis with implications for travel medicine. Travel Med Infect Dis. 2016;14(2):143-7. https://doi.org/10.1016/j. tmaid.2015.11.011 Epub 2015 Dec 18.

2. Puca E, Majko J, Puca E, Qyra E, Gega A, Pipero P. Acute encephalitis as initial presentation of leptospirosis. J Infect Dev Ctries. 2017;11(4):361-3. https://doi.org/10.3855/jidc.8613.

3. Marchiori E, Lourenço S, Setúbal S, et al. Clinical and imaging manifestations of hemorrhagic pulmonary leptospirosis: a state-of-the-art review []]. Lung. 2011;189:1-9.

4. Chavez JR, Danguilan RA, Arakama MI, et al. A case of leptospirosis with acute respiratory failure and acute kidney injury treated with simultaneous extracorporeal membrane oxygenation and haemoperfusion [J]. BMJ Case Rep. 2019;12(5):e229582. https://doi.org/10.1136/bcr-2019-229582.

5. Croda J, Neto AN, Brasi RA, et al. Leptospirosis pulmonary haemorrhage syndrome is associated with linear deposition of immunoglobulin and complement on the alveolar surface [J]. Clin Microbiol Infect. 2010;16(6): 593-9.

6. Guerrier G, D'Ortenzio E. The Jarisch-Herxheimer reaction in leptospirosis: a systematic review [J]. PLoS One. 2013;8(3):e59266.

7. Butler T. The jarisch herxheimer reaction after antibiotic treatment of spirochetal infections: a review of recent cases and our understanding of pathogenesis [J]. Am J Trop Med Hyg. 2017;96(1):46-52.

8. Vijayachari P, Sugunan AP, Singh SS, Mathur PP. Leptospirosis among the self-supporting convicts of Andaman Island during the 1920s--the first report on pulmonary haemorrhage in leptospirosis [J]? Indian J Med Res. 2015;142(1):11-22

9. Fonseka CL, Lekamwasam S. Role of Plasmapheresis and extracorporeal membrane oxygenation in the treatment of leptospirosis complicated with pulmonary hemorrhages [J]. J Trop Med. 2018;2:4520185. https://doi.org/10, 1155/2018/4520185

10. Azevedo AF, Miranda-Filho Dde B, Henriques-Filho GT, Leite A, Ximenes RA. Randomized controlled trial of pulse methyl prednisolone $\times$ placebo in treatment of pulmonaryinvolvement associated with severe leptospirosis [J]. BMC Infect Dis. 2011:11:186.

11. Gattinoni L, Vasques F, Quintel M. Use of ECMO in ARDS: does the EOLIA trial really help [J]? Crit Care. 2018;22:171.

12. Pardinas M, Mendirichaga R, Budhrani $G$, et al. Use of aminocaproic acid in combination with extracorporeal membrane oxygenation in a case of leptospirosis pulmonary hemorrhage syndrome []]. Clin Med Insights Circ Respir Pulm Med. 2017;2(11):1179548416686068. https://doi.org/10.1177/ 1179548416686068

13. Vandroux D, Chanareille P, Delmas B. Etal. Acute respiratory distress syndrome in leptospirosis [J]. J Crit Care. 2019;51:165-9.

\section{Publisher's Note}

Springer Nature remains neutral with regard to jurisdictional claims in published maps and institutional affiliations. 